\title{
Implementing Human Factors within the Design Process of Advanced Driver Assistance Systems (ADAS)
}

\author{
Boris van Waterschoot and Mascha van der Voort \\ Laboratory of design, Production and Management, University of Twente, \\ Drienerlolaan 5, 7500 AE Enschede, The Netherlands \\ $\{$ b.m. vanwaterschoot, m.c.vandervoort $\}$ autwente.nl
}

\begin{abstract}
This paper introduces our research which aims to develop a design approach for ADAS applications in which human factors (including stakeholder feedback and objective performance measures) are explicitly accounted for. Since driving is part of a complex (traffic) system, with a large number of interacting components, ADAS design is confronted with choices for which the influence on the system, and the driving performance in particular, is not immediately manifested. Therefore, providing designers with relevant feedback during the design process, about the consequences of specific choices, will increase the efficiency and safety of driver assistance systems.
\end{abstract}

Keywords: Advanced driver assistance systems, driving task, human factors engineering, interaction design, design support, automation.

\section{Introduction}

In-vehicle support systems show a rapid change in terms of sharing control with the human driver and present generation technologies are shifting their support from low-level vehicle control towards high-level driving tasks. Conventional vehicle automation (e.g., automatic gear changing or cruise control) is replaced by systems that show enhanced driving automation, i.e. systems that are able to perceive, decide and act in an appropriate manner. Adaptive cruise control (ACC) for example, shows cognitive abilities in order to maintain both required driving speed and distance from the leading vehicle; cognitive tasks which are (temporarily) taken over from the driver. As [1] already addressed, these emerging trends in vehicle automation are not only advances, but the increase of automation and shifts in task control are cause for concern as well.

An extensive amount of research deals with the problems that might arise when technology and humans have to coordinate their tasks in order to reach a common goal. Endsley made an important contribution by showing that situation awareness (SA) interacts with automation [2]. In a similar vein, Walker and colleagues [3, 4] communicated their concerns regarding the implications of the increasing amount of automation in vehicle design. The present paper introduces our research, which is directly aimed at providing advanced driver assistance systems (ADAS) design with the appropriate knowledge and insight concerning the implications of supporting technologies within the driving task. 
Currently, ADAS design is highly technology-driven, which means that new functions are added when they are feasible rather than because they are needed [5]. If ADAS design tends to focus on technique, attempting to automate whatever is possible, at least two main drawbacks arise. Firstly, since the supporting technologies are often developed independently, the overall performance of the vehicle remains unknown. Potential problems due to shared control cannot be evaluated until the actual realization of a given technology within the driver-vehicle system as a whole. Secondly, mere feasibility of technology discards the view of a joint driver-vehicle system. Although single technologies (e.g., ACC or automatic parking) can offer support for specific functions within the overall driving task, they are part of a larger system in which driver and vehicle share control in order to manoeuvre in traffic. The inclusion of dedicated support should therefore complement the overall driving task without interference. By shifting the control capabilities towards the higher level driving task, driver support systems are reducing certain requirements placed on the driver. The implications of driver support, however, are not clear-cut because improper driver support can be responsible for both mental under- and overload. Designing ADAS is therefore not only confronted with the mere implementation of advanced technologies, but should address implications for human operation as well.

The present paper asserts that scientific inquiries have already provided much insight about the influence of automation on human (cognitive) performance. Multidisciplinary fields like cognitive engineering or human factors research have put much effort in highlighting the issues that arise with the emergence of automation. However, specific solutions how to overcome unwanted implications related to automated driver support, are not readily available. For this reason, our research will be aimed at developing an adaptive design environment, in which designers are provided with the consequences of their design choices on human-system performance. This knowledge should support the implementation of technologies that complement the human driver within the overall driving task. A complementation that is intended to improve the quality of the (joint) cognitive system in terms of efficiency, safety and convenience.

The appreciation for evaluating in-vehicle systems already exists and is supported by a large body of scientific knowledge that encompasses, among others, driving behaviour, human factors and human-technology interaction. However, true knowledge about how to translate systems' evaluation into specific design improvements is still lacking. This is mainly due to the fact that the influence of additional design changes (or re-designs) on the driving behaviour remains unknown until a re-evaluation takes place. Secondly, as already mentioned, the industry of ADAS applications is highly technology driven. Which means that whether a certain technology is implemented often depends on the possibilities for such a development instead of the true need from a driver-vehicle system point of view. We argue that these approaches are not mutually exclusive, but can only be realized when ADAS designers receive feedback about the system's requirements and when the nature of potential problems are evaluated during the design process. If the influence of design choices cannot be predicted in advance, they should at least be evaluated early in the design process with short and adaptive iterations. Before addressing the main issues of ADAS design and evaluation in Chapter 4, we will first address the role of ADAS in contemporary driving and the consequences of increased automation. 


\section{Driving and Advanced Driver Assistance Systems}

In 1938 [7] driving was thought as psychologically analogous to walking or running, with the addition that driving is locomotion by means of a tool (i.e. the car). In this view, the goal of the driver is to move from one point in space to another; the destination. During this locomotion obstacles are perceived and the drivers' task is to avoid them. While driving can still be seen as an intimately merged perceptual-motor task, today's driving task is seen as considerably more than that [8] and theoretical attempts to understand the driving task are still challenging in the scientific community. Two main contributors to a changing driving task are the introduction of ADAS in modern day cars, which is (deliberately) aimed at reducing the requirements placed on the driver, and the car manufacturers' aim to make driving more comfortable.

The predominant reason for introducing in-vehicle support systems is the assumption that by supporting the driving task through increased automation, the drivers receive additional information and driver support enables them to share the overall driving task with dedicated support. Reducing the cognitive efforts placed on the driver and providing relevant information, should make driving more comfortable and safer. By equipping vehicles with sensors, navigation and motion planning the driving task is shared between human actors and the supporting assistance systems. By adding and improving cognition and control techniques, this could lead to autonomous vehicles in which the driving task is controlled by the vehicle and the responsibility is shifted towards the vehicle and its manufacturer. Although legal issues and high infrastructural demands will prevent the introduction of such autonomous vehicles in the near future, research has already provided (semi-) automated concept cars in which minimal or no intervention of human actors is required. Meanwhile, different assistance systems are already supporting the present day driver by means of sensory information (e.g., visibility aids or lane departure warnings), correction (e.g., anti-lock braking system or traction control) or even control (e.g., automatic parking).

Apart from introducing ADAS, the experience of driving changed with the assumption employed in modern day cars, that it is beneficial to reduce internal car noise [9]. According to [10] minimizing interior vehicle noise and vibration would allow passengers to "enjoy the latest advances in communications and entertainment technologies" (page 83). In accordance, [4] observed that in automotive design the level and type of feedback available to the driver, is diminishing. Although this trend (reduced interior vehicle noise and applying softer suspension) is not directly within the scope of our research, it is mentioned for two reasons: First, it is an explicit example of reduced feedback received by the modern day car driver. Reducing the noise and vibrations made by the car (generated by the engine, tires and wind), either by isolating the drivers' cockpit from external noise or through the use of absorbing techniques and materials, reduces valuable motion cues, which are used to make safety-related judgments [4]. Reduced (auditory) feedback alters drivers' speed perception, which potentially causes them to choose faster speeds, and places them at greater risk of crashing [9] because drivers' choice of driving speed has been found to be an important predictor of crash risk [11-13]. This brings us to a second reason why we mention the trend of reducing internal car noise. While reducing the level of 
internal noise is seen as a goal in designing modern cars, it shows an emerging conflict between comfort and safety. In addition, it shows a conflict with the efforts made by developing ADAS applications, because these supporting systems should increase both driver's comfort and safety. By increasing comfort through designing cars with reduced levels of internal noise, potential safety issues arise that are unwanted. While car design and the implementation of dedicated support systems should result in safe and comfortable driving, it is argued that these attempts are often considered in isolation and that comfort and safety are not necessarily values on the same coin.

Gibson and Crooks [7] described the driving task as "locomotion within an optic array" and the concept of traffic can be described as a dynamical system [14]. The driver-vehicle system, which travels within traffic, is dealing with constraints and these constraints can be defined as either limitations or abilities (affordances) but are generally investigated separately for human and machine (or artefacts). Performance issues (e.g., memory and skills or processing speed) are for the most part determined separately for user and artefact, without viewing the overall performance as a quality of the driver-vehicle system. Although the view of a unified driver-vehicle systems is generally recognized, it does not comply with most design- or evaluation research, which usually approaches human- and vehicle behaviour separately. Due to a wide range of feedback and assessment techniques, the possibilities to evaluate humanmachine systems are numerous and the development of controlled research environments (e.g., driving simulations and validated experimental designs) resulted in a large body of investigations concerning the influence of in-vehicle support on the users' behaviour $[15,16]$. Oddly enough it is either the human factor or the technology that is subject to investigation. In the development and evaluation of $\mathrm{HCI}$ environments, humans are often seen as the weakest link [17] and their cognitive and physical properties are typically explained as limitations. This stance however is arbitrary and potentially narrows the perspective of the researcher. Furthermore, this view may result in a machine centred bias, which argues that automation compensates for human inadequacies [18]. The expectation that "machines do it better" induces at least a competition between humans and technology, while they are expected to cooperate. Consequently, this section argues that the observed trend of increased automation in the driving task poses new challenges in the design of driver support.

\section{Automating Driver Support}

Due to the introduction of (semi-) automated support systems, a shift in control has taken place within the driving task [1]. This has led to three main consequences.

First of all, the increase of automation and the shift in control have led to the recognition of behavioural consequences for the driver. The out-of-the-loop problem (which can be characterized as an insufficient interaction between driver and vehicle) and the related decrease of situation awareness are typical examples of this influence $[19,2]$.

Secondly, the shift in control between driver and in-vehicle support has led to an additional shift concerning the responsibilities and the level of interaction between 'human and machine'. While in conventional driving the driver is in full control, a 

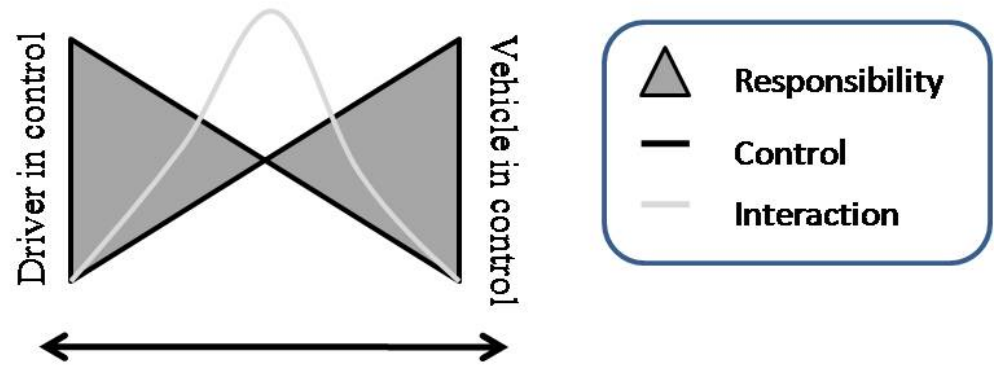

Fig. 1. Showing an idealized, although simplified picture of the relationship between driver and vehicle within the human-vehicle system (HVS). The x-axis represents the (cumulative) level of control, which corresponds linearly with the amount of responsibility within the driving task. The $y$-axis represents the level of interaction between Driver and Vehicle, which in its turn depends on the amount of control (x-axis).

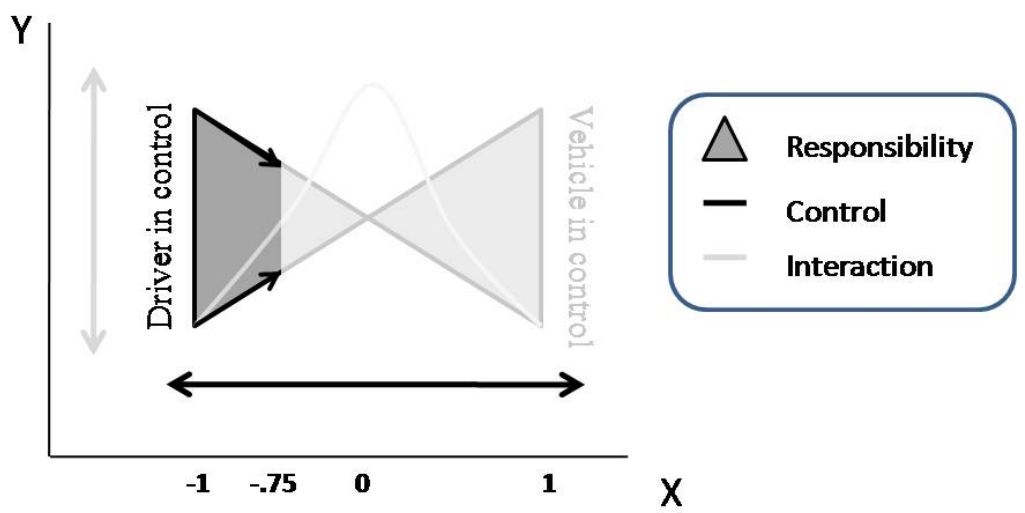

Fig. 2. An example of the interactive relationship between Responsibility and Control of the driving task

control shift occurs when the need for human intervention diminishes (theoretically, in a fully autonomous vehicle no intervention is needed). When control shifts towards the vehicle and its supporting systems, the interaction between vehicle and driver increases which potentially results in an additional shift in responsibility. Theoretically, in a fully autonomous vehicle with no human interventions (i.e. no human control in the driving task) responsibility is shifted entirely towards the vehicle and its manufacturer. Figure 1 shows a hypothetical image of this changed relationship between driver and vehicle due to the increase of automation and the shift in control. In order to exemplify the relationship between driver and vehicle, an example is presented in Figure 2. At $(X=-1)$, the driver is in full control and receives no additional information from the vehicle. Here, the amount of interaction (grey line, 
y-axis) is zero. Accordingly, the amount of driver's responsibility within the overall driving task, decreases as the amount of interaction between driver and vehicle increases. At $(\mathrm{X}=-.75)$, the driver is sharing his responsibility with the vehicle, as he's receiving additional information from the latter (e.g., driving speed, temperature, distance to preceding cars, etc.). The amount of interaction (between driver and vehicle) increased as opposed to $(X=-1)$, because the information presented by the vehicle influences the driver's behaviour. In its turn, this information is highly interdependable on the driver's behaviour, because he can act on this information accordingly (e.g., increasing speed, decreasing distance to preceding car, etc.). At (X $=0$ ), driver's and vehicle's control of the overall driving task are in an (metaphorical) equilibrium. Here, the amount of control and responsibility are equally shared, and the interaction between driver and vehicle is maximal. The expression "equilibrium" is chosen to emphasize the instability of this level of control. After all, up till today, any type of information or supporting assistance system (e.g., driving speed or adaptive cruise control, respectively) can be overruled or ignored by the driver. Therefore, the state in which the control of the driving task is equally shared between driver and vehicle (and hence, the available ADAS) remains hypothetical.

A third consequence of the increase of automation and the shift in control towards the vehicles' supporting systems is the grown appreciation in the human factors and design communities for the potential (safety) problems involved. Psychological constructs like underload and overload have been adopted within the driving context (e.g., [20]) and mental (work)load and performance issues gained increased attention. However, although these constructs are able to reveal the influence of automation on the behaviour of the human driver, no consensus exists about the optimum values of these concepts. Individual differences and (human) cognitive flexibility are two main reasons for this shortcoming.

\section{ADAS Design and Evaluation}

Regardless of the nature of the systems at stake, for ADAS designers it is important that they are provided with the impact caused by their design choices, manifested by (for instance) distraction related problems or cognitive under- and overload. Evaluation will enable the designer to improve or modify the concept according to the evaluation outcome. Human factors experts and ADAS designers try to optimize ADAS applications by using evaluation sessions and experimentally obtained information. They are confronted, however, with two main problems.

Even if close collaboration between design and human factors research is established, at least temporal disparities remain between actual design decisions and subsequent evaluation. When prototypes are evaluated (e.g., by their impact on driving behaviour) researchers receive performance outcomes and provide recommendations with which designers head back to the 'drawing board'. After applying design changes, the design process is back where it was when the first prototype was finished: not yet able to reveal its impact on the driving behaviour and its overall surplus value. Moreover, the assessment of design choices is not only concerned with the influence on the driving behaviour, but both design and evaluation are concerned with a driver-car-traffic system that changes when individual in-vehicle 
applications are added or reconfigurated. When dealing with such a complex system, ADAS design and evaluation are confronted with the difficult task to disentangle the influence of specific design choices.

A second problem concerning the evaluation of ADAS applications (and hence ADAS design in general) deals with the type of information the designers have to deal with. Psychological constructs like situation awareness and workload, which are well adapted within human factors- and psychological research, have proven their applicability in order to address problems that concern the interaction between technology or artefacts and their users. Although experimental designs and evaluations using these constructs are appreciated for their ability to account for the human factor (i.e. how does technology influences human behaviour and which behavioural effects arise when humans interact with automation?), they do not provide explicit information for the designers to hold on. The behavioural constructs and the related experimental outcomes are therefore not able to directly instruct how ADAS design should be improved.

Human factors- and design communities still lack a considerable amount of common ground [21]. For ADAS design and evaluation this has two major implications. On the one hand the technological stance often puts human factors professionals in a difficult position, because when consulted, they have to convince engineers of the importance of human centred design. On the other hand the evaluation considering human factors is confronted with the difficult task to translate its results (the influence of in-vehicle support on driver- and vehicle behaviour) into specific design considerations, which presents ADAS designers with the difficulty of accounting for human factors in accordance with the systems' performance and the preferred outcome, including stakeholders' preferences.

\section{Conclusions and Future Work}

In this paper we argue that present-day driving and the design of automated driver support has three main issues to deal with.

In general ADAS design has to deal with a biased technological viewpoint. Developers ask themselves if and how new technologies can be implemented [5]. During this process human factors professionals evaluate the assistance system through the behavioural impact on the driving behaviour of the user or the performance of the vehicle. Psychological constructs used for this evaluation are for example situation awareness and workload. This type of research provides a clear view of the influence or impact on the driving behaviour and (re)design recommendations can be made according to this research. The behavioural and physiological measurements used for evaluation, however, do not directly relate to or 'produce' design suggestions. This means that a conversion from evaluation results into design solutions is missing. In addition, while the measurements ordinarily used for evaluation are able to present the influence of isolated and controlled independent variables on the driving behaviour (typically represented by SA, workload or other psychological measures) they do not relate to the (entire) driver-vehicle system, let alone to the influence on the traffic system. In our view, design should therefore take account of the needs, competencies and limitations of the joined driver-vehicle system. 
Secondly, in the development process of ADAS applications by the automotive industry much effort is spent to present prototypes before evaluating them. This iterative process can take a while and is reflected by high developmental costs. If experts from different disciplines (e.g., designers, engineers, human factors practitioners) would test the design at an earlier stage, the performance and impact of specific design choices could be observed more rapidly. This would enable them to apply design improvements during the design process. Improvements can be made by using, for example, VR simulations, user-experiences, performance measures and expert collaboration. In the present paper we argue that a distinct, straightforward and controlled design environment, which accounts for human factors and at the same time enables designers to improve their designs with unambiguous design solutions, is still lacking. This could be overcome by early involvement of human factors knowledge during the design process.

Finally, the increase of automation and the shift of control in the driving task are reason for concern and raises the question of how to deal with the consequences and how to prevent unwanted and unforeseen effects. A vast body of scientific research allows us to evaluate the consequences of automation and control shifts, but ready to use solutions (that are applicable and reliable for each configuration) are not available. It is agreed in a large body of research that because automation shifts control and potentially influences driver behaviour or modifies the driving task, safety can be jeopardized [22-26]. However, no consensus exists about how to implement automation in order to optimize driver support. Perhaps Norman [21] is quite revealing when he asserts that "automation always looks good on paper. Sometimes you need real people". At least the (automated) support and driver system should be in 'balance' and complement each other. While technological innovations can be promising and entail potential surplus value, they cannot disregard the actual and true human needs that are represented by 'the human factor' that keeps the driver in the loop. Optimizing driver support is an endeavour that will remain a current affair until we can seat ourselves in the first commercially available autonomous vehicle and human intervention is minimal.

To summarize, the aim of our study is to improve the design process of advanced driver assistance systems by developing a new design approach. We introduced our research by discussing some major issues concerning advanced driver assistance, its design and the implementation of automation. In our view, a new design approach should:

- Be able to take advantage of both human abilities (i.e. human factors) and technological possibilities.

- View the driving task as being part of a joint driver-vehicle system.

- Support the design process with expert knowledge about the systems' needs, expectations and abilities.

- Be able to identify performance (i.e. facilitate systems' evaluation).

- Translate evaluation into design solution or -suggestion.

In order to realize a surplus value, the new design approach will implement human factors knowledge during the design process. In line with [27] the design environment therefore enables, at least, early stakeholder involvement. Next steps towards an integration of human factors and ADAS design are twofold. On the one hand 
subsequent research will consist of modelling the relation between driving functions and the related systems' behaviour. One the other hand we will examine the integration of problem, solution and assessment within the context of driver support. In addition, we will explore the applicability of (existing) behavioural measures, having the strength to serve the translation between evaluation and design.

\section{Acknowledgement}

This research is part of the knowledge centre AIDA, a collaboration between TNO and the University of Twente.

\section{References}

1. Young, M.S., Stanton, N.A., Harris, D.: Driving automation: learning from aviation about design philosophies. International Journal of Vehicle Design 45, 323-338 (2007)

2. Endsley, M.R., Kiris, E.: The out-of-the-loop performance problem and level of control in automation. Human factors 37, 381-394 (1995)

3. Walker, G.H., Stanton, N.A., Young, M.S.: Where Is Computing Driving Cars? International Journal of Human-Computer Interaction 13, 203-229 (2001)

4. Walker, G.H., Stanton, N.A., Young, M.S.: The ironies of vehicle feedback in car design. Ergonomics 49, 161-179 (2006)

5. Hollnagel, E.: A function-centred approach to joint driver-vehicle system design. Cognition, Technology \& Work 8, 169-173 (2006)

6. Young, M.S., Stanton, N.A.: Malleable Attentional Resources Theory: A New Explanation for the Effects of Mental Underload on Performance. Human Factors 44, 365-375 (2002)

7. Gibson, J.J., Crooks, L.E.: A Theoretical Field-Analysis of Automobile-Driving. The American Journal of Psychology 51, 453-471 (1938)

8. Groeger, J.A.: Understanding Driving: Applying Cognitive Psychology to a complex everyday task. Psychology Press, Hove (2000)

9. Horswill, M.S., Plooy, A.M.: Auditory feedback influences perceived driving speeds. Perception 37, 1037-1043 (2008)

10. Trainham, J.: Quiter rides. Automotive Engineering International 113, 83 (2005)

11. Wasielewski, P.: Speed as a measure of driver risk: observed speeds versus driver and vehicle characteristics. Accident Analysis and Prevention 16, 89-103 (1984)

12. Horswill, M.S., McKenna, F.P.: The development, validation, and application of a videobased technique for measuring an everyday risk-taking behaviour: drivers' speed choice. Journal of Applied Psychology 84, 977-985 (1999)

13. Evans, L.: Traffic Safety. Science Serving Society, Bloomfield Hills (2004)

14. Cantarella, G.E., Cascetta, E.: Dynamic Processes and Equilibrium in Transportation Networks: Towards a Unifying Theory. Transportation Science 29, 305-329 (1995)

15. Stanton, N.A., Young, M.S.: Vehicle automation and driving performance. Ergonomics 41, 1014-1028 (1998)

16. Wierwille, W.W.: Demands on driver resources associated with introducing advanced technology into the vehicle. Transportation Research Part C: Emerging Technologies 1, 133-142 (1993)

17. Flach, J.M., Hoffman, R.R.: The Limitations of Limitations. IEEE Intelligent Systems 18, 94-96 (2003) 
18. Norman, D.A.: Things That Make Us Smart. Addison-Wesley, Boston (1993)

19. Endsley, M.R.: Toward a Theory of Situation Awareness in Dynamic Systems. Human Factors 37, 32-64 (1995)

20. Walker, G.H., Stanton, N.A., Young, M.S.: Feedback and driver situation awareness (SA): A comparison of SA measures and contexts. Transportation Research Part F: Traffic Psychology and Behaviour 11, 282-299 (2008)

21. Norman, D.A.: The Design of Future Things. Basic books, New York (2007)

22. Sheridan, T.B.: Human Factors of Driver-Vehicle Interaction in the IVHS Environment. Publication No. NHTSA/MIT, DOT-HS-807-737, National Highway Traffic Safety Administration, Department of Transportation, Washington, DC (1991)

23. Hancock, P.A., Parasuraman, R.: Human factors and safety in the design of intelligent vehicle-highway systems (IVHS). Journal of Safety Research 23, 181-198 (1992)

24. Michon, J.A.: Generic Intelligent Driver Support. A comprehensive report on GIDS. Taylor \& Francis, London (1993)

25. Verwey, W.B.: Further evidence for benefits of verbal route guidance instructions over symbolic spatial guidance instructions. In: Reekie, D.H.M. (ed.) Proceedings of the Vehicle Navigation and Information Systems Conference (VNIS 1993), pp. 227-231. IEEE, Toronto (1993)

26. Lansdown, T.C., Brook-Carter, N., Kersloot, T.: Distraction from Multiple In-Vehicle Secondary Tasks: Vehicle Performance and Mental Workload Implications. Ergonomics 47, 91-104 (2004)

27. van der Voort, M.C., Tideman, M.: Combining Scenarios and Virtual Reality into a New Approach to Including Users in Product Design Processes. Journal of Design Research (in press) 